Short communication

\title{
A panel of microsatellite markers to discriminate and study interactions between Haemonchus contortus and Haemonchus placei
}

\author{
Michelle C. Santos $^{\mathrm{a}, *}$, Elizabeth Redman ${ }^{\mathrm{b}}$, Mônica R.V. Amarante ${ }^{\mathrm{a}}$, John S. Gilleard ${ }^{\mathrm{b}}$, \\ Alessandro F.T. Amarante ${ }^{\mathrm{a}}$ \\ ${ }^{a}$ Universidade Estadual Paulista (UNESP), Departamento de Parasitologia, Instituto de Biociências; Botucatu - SP, Brazil \\ ${ }^{\mathrm{b}}$ University of Calgary, Department of Comparative Biology and Experimental Medicine, Faculty of Veterinary Medicine; Calgary, Alberta, Canada
}

\section{A R T I C L E I N F O}

\section{Keywords:}

Gastrointestinal nematode

Haemonchus spp

Microsatellites

Molecular biology

Ruminant

\begin{abstract}
A B S T R A C T
Haemonchus contortus and Haemonchus placei are two closely related economically important parasites of ruminants. Their close morphological similarity, common occurrence as co-infections and ability to hybridize makes definitive diagnosis and epidemiological studies in field populations challenging. In this paper, we describe the development of a panel of microsatellite markers that can be used to discriminate and study the genetics of these two parasite species in co-infections and mixed field populations. We have identified two additional microsatellites (Hp52 and Hp53), in addition to three previously reported microsatellites (Hcms3561, Hcms53265 and Hcms36) that have a discrete set of alleles between the two species. Multilocus genotyping of worms with this 5 marker panel from 3 geographically diverse $H$. placei isolates and 4 geographically diverse $H$. contortus populations allows unambiguous species assignment of individual worms. This panel of markers should provide a valuable resource in studying the biology and epidemiology of these important ruminant parasite species in the field.
\end{abstract}

\section{Introduction}

Haemonchus contortus and Haemonchus placei species are economically important gastrointestinal nematodes of ruminants which cause serious negative impacts on animal welfare and productivity (O'Connor et al., 2006; van Dijk et al., 2010). These two species are extremely closely related and can hybridize to produce viable $\mathrm{F}_{1}$ progeny which are fertile upon mating with parental species (Le Jambre, 1979, 1981). Although sheep and cattle are the respective preferred hosts, these parasites often co-infect the alternate host species and co-infections are common, particularly when cattle and sheep share the same pastures (Amarante et al., 1997; Chaudhry et al., 2015).

Attempts to use morphology and morphometrics to distinguish Haemonchus species, have encountered considerable issues with overlapping measurement ranges (Santos et al., 2014). Molecular techniques offer advantages over morphology for the identification of parasites, their genetic characterization, the isolation and characterization of expressed genes, and the detection of drug resistance and mutations (Gasser, 1999; Gasser et al., 2016). Species-specific microsatellite genotyping of populations of gastro-intestinal worms is well-established in the literature and represents a convenient technique to characterize strains of parasitic nematodes such as $H$. contortus and Teladorsagia circumcincta (Hoekstra et al., 1997; Redman et al., 2008a). The use of microsatellites to discriminate between $H$. contortus and $H$. placei individual worms and also identify hybrid worms was first described using a panel of three markers on parasite populations from Pakistan and India (Chaudhry et al., 2015). In this study, we build on this previous work by characterising two additional markers and validating the discriminatory power of the expanded marker panel on $H$. placei and $H$. contortus isolates from more diverse geographical regions.

\section{Materials and methods}

Our initial screening and characterization of the previous and new markers was performed on a $H$. contortus and a $H$. placei isolate from Sao Paulo, Brazil. The H. contortus isolate (SpHco2) was isolated from larvae from two male lambs raised on a farm in Sao Paulo state, Brazil naturally infected with gastrointestinal nematodes (GIN) in 2006, while the $H$. placei isolate (SpHpl1) larvae were isolated from a bovine naturally infected with GIN in Sao Paulo state in 2005. 25,000 infective larvae (L3s) of each isolate were used to infect a donor sheep $(H$. contortus, SpHco2) and a calf (H. placei, SpHpl1) to generate adult worms for this study. These donor animals (sheep and calf) were maintained indoors and, before being infected with the isolates, received monepantel

\footnotetext{
* Corresponding author.

E-mail address: michelle@ibb.unesp.br (M.C. Santos)
} 
(A)



8

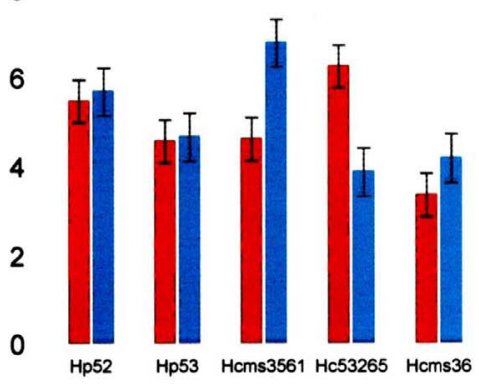

(D)

Hcms 3561
(B)

$$
H_{e} \text { (unbiased) }
$$

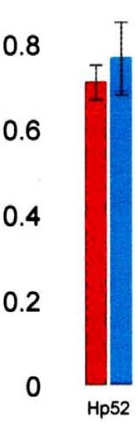

(E)

0.8

0.6

$$
0.6
$$

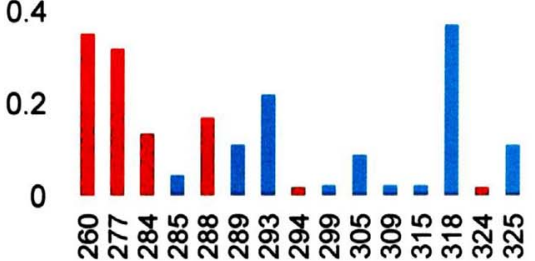

0.4

0.2

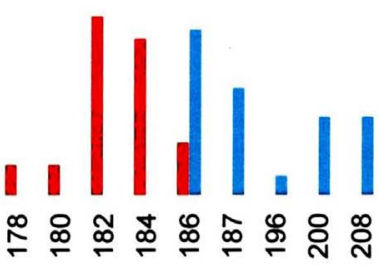

(C)

Estimated mean unique allele richness (rarefaction, genes $=20$ )
0.4

0.8

(G)

Hp52

0.8

0.6

0.4

0.2

0
(H)

Hcms53265

0.8

0.6

0.4

0.2

0



8

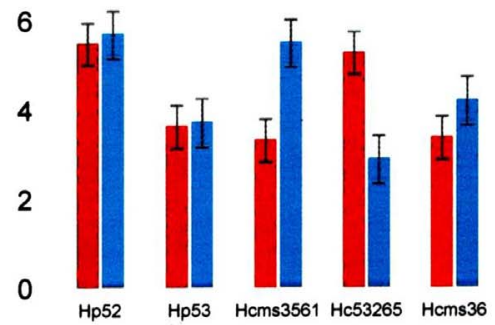

(F)

Hcms36
0.8

0.6

0.4

0

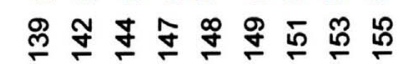


Table 1

Summary statistics and AMOVA analysis for genotype data for the five microsatellites (Hcms3561, Hp53, Hp52, Hcms36 and Hcms53265) of Haemonchus contortus (SpHco2) and Haemonchus placei (SpHpl1) populations.

\begin{tabular}{|c|c|c|c|c|c|}
\hline Loci & Hcms3561 & Hp53 & Hp52 & Hcms36 & Hcms53265 \\
\hline \multicolumn{6}{|l|}{$\begin{array}{l}\text { Haemonchus } \\
\text { contortus }\end{array}$} \\
\hline Genotypes & 30 & 30 & 30 & 30 & 30 \\
\hline $\mathrm{Na}$ & 6 & 5 & 7 & 4 & 9 \\
\hline$H_{\mathrm{o}}$ & 0.76667 & 0.66667 & 0.63333 & 0.53333 & 0.73333 \\
\hline$H_{\mathrm{e}}$ & 0.74350 & 0.70678 & 0.71299 & 0.57175 & 0.82373 \\
\hline $\mathrm{p}$-value & 0.14048 & 0.84859 & 0.16947 & 0.43332 & 0.31930 \\
\hline s.d. & 0.00025 & 0.00036 & 0.00037 & 0.00045 & 0.00047 \\
\hline \multicolumn{6}{|l|}{ Haemonchus placei } \\
\hline Genotypes & 23 & 23 & 19 & 19 & 18 \\
\hline $\mathrm{Na}$ & 9 & 5 & 6 & 5 & 5 \\
\hline$H_{\mathrm{o}}$ & 0.47826 & 0.56522 & 0.63158 & 0.52632 & 0.27778 \\
\hline$H_{\mathrm{e}}$ & 0.79903 & 0.76039 & 0.76956 & 0.48080 & 0.38889 \\
\hline $\mathrm{p}$-value & $0.00003^{*}$ & 0.06515 & 0.22626 & 0.47369 & $0.02102^{*}$ \\
\hline s.d. & 0.00001 & 0.00024 & 0.00045 & 0.00052 & 0.00011 \\
\hline \multicolumn{6}{|l|}{ AMOVA: } \\
\hline $\begin{array}{c}\text { Variation among } \\
\text { populations }\end{array}$ & 19.65 & 24.42 & 25.83 & 46.85 & 36.01 \\
\hline $\begin{array}{l}\text { \% Variation among } \\
\text { individuals } \\
\text { within } \\
\text { populations }\end{array}$ & 13.41 & 11.30 & 10.50 & 0.62 & 9.78 \\
\hline $\begin{array}{l}\% \text { Variation within } \\
\text { individuals }\end{array}$ & 66.94 & 64.28 & 63.67 & 52.54 & 54.21 \\
\hline \multicolumn{6}{|l|}{ Fixation indices: } \\
\hline $\mathrm{F}_{I S}$ & 0.17 & 0.15 & 0.14 & 0.01 & 0.15 \\
\hline$p$-value & 0.01271 & 0.05181 & 0.05376 & 0.51320 & 0.03226 \\
\hline $\mathrm{F}_{S T}$ & 0.20 & 0.24 & 0.26 & 0.47 & 0.36 \\
\hline$p$-value & 0 & 0 & 0 & 0 & 0 \\
\hline $\mathrm{F}_{I T}$ & 0.33 & 0.36 & 0.36 & 0.47 & 0.46 \\
\hline$p$-value & 0 & 0 & 0 & 0 & 0 \\
\hline
\end{tabular}

Na: number of alleles, $H_{\mathrm{o}}$ : observed heterozygosity and $H_{\mathrm{e}}$ : expected heterozygosity.

$* p$-value $<0.05$ indicate a significant deviation from Hardy-Weinberg equilibrium. Global AMOVA results: average F-Statistics over all loci for fixation indices: inbreeding coefficient $\left(\mathrm{F}_{I S}\right)$ : 0.12080 , fixation index $\left(\mathrm{F}_{S T}\right): 0.30763$ and overall fixation index $\left(\mathrm{F}_{I T}\right)$ : 0.39819 .

water and grass hay (Cynodon dactylon cv. Tifton 85 ad libitum) purchased from a farm with no ruminants, avoiding risks of food contamination by nematode L3. Faecal cultures were performed separately for the production of L3. The species identity were confirmed based on the length of the sheath tail extension using an ocular micrometer (Zeiss ${ }^{\circ}$ ), $H$. placei larvae usually have a sheath tail longer than $85 \mu \mathrm{m}$ and $H$. contortus a sheath tail shorter than $85 \mu \mathrm{m}$ (Santos et al., 2014). The donor animals were euthanized 106 and 161 days post-infection (DPI), for the sheep and calf respectively and adult worms recovered and immediately fixed in $70 \%$ ethanol. Adult male worms were morphologically identified, in accordance to the Manual of Veterinary Parasitology Laboratory Techniques using male tail and spicule morphology (MAFF, 1986), prior to DNA preparation. Individual adult males $(\mathrm{n}=30$ for $\mathrm{SpHco} 2$ and $\mathrm{n}=23$ for SpHpl1) were randomly chosen for detailed molecular analysis and following sharp dissection DNA lysates were prepared from the anterior half using previously described methods (Redman et al., 2008a). The rDNA ITS-2 sequence of SpHpl1 and SpHco2 adult worms were generated to confirm their identity, based on SNPs differences between the Haemonchus species (Chaudhry et al., 2015).

In addition to these Brazilian strains, another three $H$. contortus and two $H$. placei strains previously archived in the laboratory were used in this study. They originated from a wide range of geographical regions to explore the ability of the markers to discriminate species in different global genetic populations of these species. The $H$. contortus strains used were MHco3(ISE) (Scotland), MHco4(WRS) (S. Africa), MHco10(CAVR) (Australia). The $H$. placei strains were RAHpl2 (Argentina) and MHpl1 (Australia).

\section{Microsatellites}

To date only three microsatellite loci (Hcms36, Hcms3561 and Hcms53265) have been identified as population genetic loci for both Haemonchus contortus and Haemonchus placei (Chaudhry et al., 2015). These loci can be consistently amplified from both species, are polymorphic and yield distinct allele profiles. To expand this panel of microsatellite loci two different strategies were attempted. The first was to screen microsatellite loci already identified for $H$. contortus, for their ability to amplify H. placei. Seven Haemonchus contortus microsatellites located on the X-chromosome (Redman et al., 2008b) were tested for their ability to amplify Haemonchus placei individuals. Two (HcmsX142 and HcmsX182) of the seven loci, showed potential and were selected for further investigation. The second approach was to bioinformatically identify potential microsatellites that could, theoretically be amplified from both $H$. placei and $H$. contortus, based on their sequence alignments. Of the twelve loci that were identified in this manner, five loci (Hp102, Hp43, Hp35, Hp52 and Hp53) produced distinct allele ranges for the different species and were consequently chosen for further study (data not shown).

These seven microsatellite markers (HcmsX142, HcmsX182, Hp102, Hp43, Hp35, Hp52 and Hp53), that had shown potential for amplifying both species, were used to genotype the 23 and 30 individual adult worms from $H$. contortus (SpHco2) and H. placei (SpHpl1) respectively. Of these two loci, Hp52 and Hp53, consistently produced distinct, easily binned amplicons and were therefore chosen to add to the previously described markers (Hcms3561, Hcms36, Hcms53265) (data not shown).

The characteristics and primers pairs for Hcms3561, Hcms36, Hcms53265 have been previously described (Chaudhry et al., 2015). Details of the two new markers are as follows: Hp52 has a repeat sequence of $[\mathrm{AAAGA}]_{5}$ AAAGG AAAGN $[\mathrm{AAAGA}]_{9}$ and was amplified using primers 5'-GAGTGAGACATCGTAATCCA- $3^{\prime}$ and 5'-GGTATCAAACTACTACTTGTC- $3^{\prime}$ an annealing temperature of $54{ }^{\circ} \mathrm{C}$ and allele size range (340-470 bp). Hp53 has repeat sequence of $[$ ACAT] 7 NACAT $[\mathrm{ACAT}]_{7}$ and was amplified using primers $5^{\prime}$-TCGACTCTCACCCGAATACA- $3^{\prime}$ and 5'-TTTGTGGCAGTTGGCCGTT-3'at an annealing temperature of $54{ }^{\circ} \mathrm{C}$ and allele size range of $170-220 \mathrm{bp}$. PCR was performed in $20 \mu \mathrm{L}$ using Qiagen multiplex PCR kit (QIAGEN, Hilden, Germany) and $0.1 \mu \mathrm{M}$ forward and reverse primers and $2 \mu \mathrm{L}$ of lysate DNA. Thermocycling conditions evaluated were $95{ }^{\circ} \mathrm{C}$ for $15 \mathrm{~min}$ followed by 40
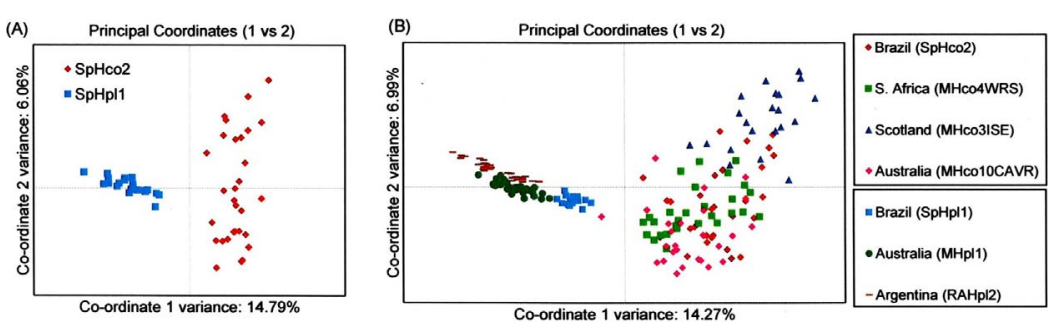

Fig. 2. Principle Component Analysis (PCA) of individual adult worm multi-locus genotypes based on five loci (Hcms53265, Hcms3561, Hp52, $\mathrm{Hp} 53$ and Hcms36). Panel A compares the SpHcol (Haemonchus contortus) and SpHpl2 (Haemonchus placei) strains from Sao Paulo Satate, Brazil. Panel B compares geographically diverse isolates $H$. contorus and $H$. placei strains (4 isolates of $H$. contortus and 3 isolates of $H$. placei) including the two strains from Brazil ( $\mathrm{SpHco} 2$ and SpHpl1). The country of origin for the strains is indicated in the legend. PCA was performed in GenAlEx version 6.5 with individual genotypes of the markers preserved; therefore each data point represents an individual worm. The percentage of variation explained by the first two coordinates is shown in the $\mathrm{X}$ - and $\mathrm{Y}$-axes of the 
cycles of $94{ }^{\circ} \mathrm{C}$ for $30 \mathrm{~s}, 54^{\circ} \mathrm{C}$ for $90 \mathrm{~s}$ and $72{ }^{\circ} \mathrm{C}$ for $30 \mathrm{~s}$ with a single final extension cycle of $72{ }^{\circ} \mathrm{C}$ for $15 \mathrm{~min}$. The forward primer of each microsatellite primer pair was 5'end labelled with 6-FAM and HEX fluorescent dyes (MWG) and electrophoresed. Microsatellite PCR products by capillary electrophoresis were performed using GeneScan ROX 400 internal size standard was used on the ABI Prism 3100 genetic analyser (Applied Biosystems, USA ${ }^{\circ}$ ). Individual chromatograms were analysed using Peak Scanner ${ }^{\mathrm{TM}}$ software to determine the genotypes of each sample and each of these five molecular markers produced distinct genotypes with a single or double peak (homozygous or heterozygous) as predictable in a diploid organism.

The unbiased expected and observed heterozygosity $\left(\mathrm{H}_{\mathrm{E}}\right.$ and $\left.\mathrm{H}_{\mathrm{o}}\right)$ were calculated with Arlequin 3.5.2. (Excoffier and Lischer, 2010). Allele richness $\left(\mathrm{A}_{\mathrm{c}}\right)$ and unique allele richness $\left(\mathrm{Au}_{\mathrm{c}}\right)$ for each locus were estimated with rarefaction (genes $=20$ ) using HP-Rare 1.1 (Kalinowski, 2005) to correct for unequal sample sizes. Differences in these measures of genetic diversity between populations were tested for statistical significance using a Wilcoxon rank sum test (Wilcoxon, 1945). Correction for variation in sample sizes was achieved by using the unbiased expected heterozygosity $\left(\mathrm{H}_{\text {Eunbias }}\right)$ and by estimating corrected allele richness values $\mathrm{A}_{c}$. In Arlequin 3.5.2 (Excoffier and Lischer, 2010) Guo and Thompson (1992) exact test was used to statistically evaluate deviations from Hardy-Weinberg equilibrium using a Markov chain (10000 steps, $p=0.05$ ) for all loci/population combinations. Partition of microsatellite diversity between and within populations was estimated through an analysis of molecular variance AMOVA with the data defined as 'standard' rather than 'microsatellite', as loci did not necessarily adhere to the stepwise mutation model. Pairwise $\mathrm{F}_{\mathrm{ST}}$ values were calculated and significance testing was undertaken by random permutation in Arlequin 3.5.2. (Excoffier and Lischer, 2010). PCA was performed in GenAlEx version 6.5 (Peakall and Smouse, 2006, 2012) with individual genotypes of the markers preserved; therefore each data point represents an individual worm.

\section{Results}

The mean allele richness was 5.05 for SpHpl1 and 4.86 for SpHco2 (Fig. 1A) and the mean expected heterozygosity was 0.6397 ( \pm SD 0.19038 ) for SpHpl1 and 0.71175 ( \pm SD 0.09107) for SpHco2 (Fig. 1B). There were no statistically significant differences in the overall genetic diversity, between the two species.

For the SpHpl1 strain, there were significant differences between observed $\left(H_{\mathrm{o}}\right)$ and expected $\left(H_{\mathrm{e}}\right)$ heterozygosity in two of the five loci; Hcms3561 $\left(H_{\mathrm{o}} / H_{\mathrm{e}} \quad 0.47826 / 0.79903,\right)$ and Hcms53265 $\left(H_{\mathrm{o}} / H_{\mathrm{e}}\right.$ $0.27778 / 0.38889)$. For $\mathrm{SpHco} 2$, there was no statistical differences $(p>0.05)$ in the other five loci tested (Table 1).

Both strains had a high number of unique alleles (Fig. 1C, mean unique allele richness, $A_{u}$ ). The allele ranges generated for the two species at these five loci were distinct (Fig. 1D-H), except the allele $186 \mathrm{bp}$ (loci Hp53) that amplified from both species. This was reflected in a high pairwise $\mathrm{F}_{\mathrm{ST}}$ estimate of 0.2922 . Multilocus genotypes of the Brazilian H. contortus (SpHco2) $\mathrm{n}=30$ and H. placei (SpHpl1) $\mathrm{n}=23$ was showed in separately clusters on PCA plots (Fig. 2A). In addition, between 20 and 35 worms from additional $H$. placei isolates (RAHpl2 and MHpl1) and H. contortus isolates (MHco3(ISE), MHco4(WRS) and MHco10 (CAVR)) were genotyped (Fig. 2B). All three H. placei isolates clustered together and separately from all four $H$. contortus isolates on the PCA plots of the multilocus genotypes (Fig. 2, Panel B).

\section{Discussion}

Both strains had a high number of unique alleles indicating the potential power of these loci to discriminate between these two species. In fact there was only one allele out of all five loci that was amplified from both species; Hp53 allele $186 \mathrm{bp}$. Consequently the allele ranges generated for the two species at these five loci were distinct.
Multilocus genotypes of the SpHco2 (H. contortus) and SpHpl1 $(H$. placei) isolates formed completely separate clusters on PCA plots. In order to further test the ability of this five marker panel to unambiguously discriminate between $H$. contortus and $H$. placei two additional $H$. placei isolates; RAHpl2 and MHpl1 and three additional $H$. contortus isolates; MHco3(ISE), MHco4(WRS) and MHco10(CAVR) were genotyped. The $H$. contortus strains have been morphologically characterized and passaged for many years and the origins of these strains are geographically diverse; MHco3(ISE) Scotland, MHco4(WRS) South Africa, MHco10(CAVR) Australia (Redman et al., 2008a). The H. placei isolates are also geographically diverse; RAHpl2 is a field isolate derived from a calf on a farm in Rosario, Argentina and morphologically identified as H. placei (Dr. J. Cabaret, personal communication), MHpl1 is a morphologically characterized $H$. placei isolate originally derived from Australia (Le Jambre, 1979) and which has now been passaged by experimental infection at the Moredun Institute (Edinbugh, Scotland) for many years.

In conclusion, we describe a panel of five microsatellite markers (Hcms36, Hcms3561, Hp53, Hp52 and Hcms53265) that are able to unambiguously discriminate between individual worms of $H$. contortus and $H$. placei across very geographically diverse isolates.

\section{Conflict of interest statement}

The authors declare no conflict of interest.

\section{Acknowledgements}

This study was supported by FAPESP (São Paulo Research Foundation) Grant number 14/02305-6. Michelle C. Santos received financial support from FAPESP (Grant number 12/23941-2 and 15/ 12900-1) and Alessandro F. T. Amarante received support from National Council for Scientific and Technological Development (CNPq). We are grateful to NSERC-CREAT HPI. The trial was approved and conducted in accordance with the experimental protocol approved by the local Ethics Committee (protocol number 449-CEEA) of Universidade Estadual Paulista (UNESP), Institute of Biosciences, Botucatu, Brazil.

\section{References}

Amarante, A.F.T., Bangola Junior, J., Amarante, M.R.V., Barbosa, M.A., 1997. Host specificity of sheep and cattle nematodes in São Paulo state. Brazil. Vet. Parasitol. 73, 89-104.

Chaudhry, U., Redman, E.M., Abbas, M., Muthusamy, R., Ashraf, K., Gilleard, J.S., 2015. Genetic evidence for hybridisation between Haemonchus contortus and Haemonchus placei in natural field populations and its implications for interspecies transmission of anthelmintic resistance. Int. J. Parasitol. 45, 149-159.

Excoffier, L., Lischer, H.E.L., 2010. Arlequin suite ver 3.5: A new series of programs to perform population analyses under Linux and Windows. Mol. Ecol. Res. 10, 564-567.

Gasser, R.B., Schwarz, E.M., Korhonen, P.K., Young, N.D., 2016. Understanding Haemonchus contortus better through genomics and transcriptomics. Adv. Parasitol. 94, 519-567.

Gasser, R.B., 1999. PCR-based technology in veterinary parasitology. Vet. Parasitol. 84 299-258.

Guo, S.W., Thompson, E.A., 1992. Performing the exact test of Hardy-Weinberg proportion for multiple alleles. Biometrics 48, 361-372.

Hoekstra, R., Criado-Fornelio, A., Fakkeldij, J., Bergman, J., Roos, M.H., 1997. Microsatellites of the parasitic nematode Haemonchus contortus: polymorphism and linkage with a direct repeat. Mol. Biochem. Parasitol. 89, 97-107.

Kalinowski, S.T., 2005. HP-Rare: a computer program for performing rarefaction on measures of allelic diversity. Mol. Ecol. Notes 5, 187-189.

Le Jambre, L.F., 1979. Hybridization studies of Haemonchus contortus (Rudolphi, 1803) and H. placei (Place, 1893) (Nematoda: trichostrongylidae). Int. J. Parasitol. 9, 455-463.

Le Jambre, L.F., 1981. Hybridization of australian haemonchus placei (Place, 1893), haemonchus contortus cayugensis (Das \& whitlock, 1960) and haemonchus contortus (Rudolphi, 1803) from louisiana. Int. J. Parasitol. 11, 323-330.

MAFF,, 1986. Manual of Veterinary Parasitology Laboratory Techniques. HMSO, London.

O'Connor, L.J., Walkden-Brown, S.W., Kahn, L.P., 2006. Ecology of the free-living stages of major trichostrongylid parasites of sheep. Vet. Parasitol. 142, 1-15.

Peakall, R., Smouse, P.E., 2006. GENALEX 6: genetic analysis in Excel: population genetic software for teaching and research. Mol. Ecol. Notes 6, 288-295. 
Peakall, R., Smouse, P.E., 2012. GenAlEx 6.5: genetic analysis in Excel. Population genetic software for teaching and research-an update. Bioinformatics 28, 2537-2539.

Redman, E., Packard, E., Grillo, V., Smith, J., Jackson, F., Gilleard, J.S., 2008a.

Microsatellite analysis reveals marked genetic differentiation between Haemonchus contortus laboratory isolates and providesa rapid system of genetic fingerprinting. Int. J. Parasitol. 38, 111-122.

Redman, E., Grillo, V., Saunders, G., Packard, E., Jackson, F., Berriman, M., Gilleard, J.S., 2008b. Genetics of mating and sex determination in the parasitic nematode Haemonchus contortus. Genetics 180, 1877-1887.
Santos, M.C., Amarante, M.R.V., Silva, M.R.L., Amarante, A.F.T., 2014. Differentiation of Haemonchus placei from Haemonchus contortus by PCR and by morphometrics of adult parasites and third stage larvae. Rev. Bras. Parasitol. Vet. 23, 495-500.

Manual Para Diagnóstico Das Helmintoses De Ruminantes. In: Ueno, H., Gonçalves, P.C. (Eds.), 4 ed. Japan International Cooperation Agency, Tokyo, pp. 143.

van Dijk, J., Sargison, N.D., Kenyon, F., Skuce, P.J., 2010. Climate change and infectious disease: helminthological challenges to farmed ruminants in temperate regions. Animal 4, 377-392.

Wilcoxon, F., 1945. Individual comparisons by ranking methods. Biometrics 1, 80-83. 